\title{
Estabilidade de cor do cimento de ionômero de vidro em soluções corantes: revisão integrativa
}

\author{
Color stability of glass ionomer cement in coloring solutions: integrative review \\ Estabilidad del color del cemento de ionómero de vidrio en soluciones colorantes: revisión \\ integradora
}

Recebido: 26/01/2022 | Revisado: 03/02/2022 | Aceito: 05/02/2022 | Publicado: 09/02/2022

\author{
Lívia Azevedo de Oliveira \\ ORCID: https://orcid.org/ 0000-0002-1468-0858 \\ Universidade do Estado do Rio Grande do Norte, Brasil \\ E-mail: liviaoliveiraze@ hotmail.com \\ Thaís Araújo dos Santos \\ ORCID: https://orcid.org/ 0000-0002-7833-0363 \\ Universidade do Estado do Rio Grande do Norte, Brasil \\ E-mail: thais.araujo123@hotmail.com \\ Fabianna da Conceição Dantas de Medeiros \\ ORCID: https://orcid.org/0000-0002-0169-4898 \\ Universidade do Estado do Rio Grande do Norte, Brasil \\ E-mail: fabianna.cdm@gmail.com
}

\begin{abstract}
Resumo
Introdução: O cimento de ionômero de vidro (CIV) apresenta diversas indicações na Odontologia, devido às suas propriedades de biocompatibilidade, liberação de flúor e adesão aos tecidos dentários. Objetivo: Analisar a estabilidade de cor do CIV convencional quando imerso em soluções corantes a partir de dados obtidos na literatura. Metodologia: Trata-se de uma revisão integrativa da literatura realizada através de buscas nas bases de dados PubMed, LILACS, SciELO e Cochrane Library, nas quais utilizaram-se os descritores: "glass ionomer cement", "cimentos de ionômeros de vidro", "color", "discoloration", "pigmentação" e "cor". Os critérios de elegibilidade incluíram estudos que avaliaram a estabilidade de cor de CIVs convencionais, artigos completos e disponíveis para download, além de textos publicados nos idiomas inglês, português e espanhol. Foram englobados ensaios clínicos, estudos laboratoriais e estudos in situ publicados nos últimos 25 anos. Resultados: Após análise dos artigos, foram incluídos na revisão 14 estudos. De maneira predominante, foi relatada a susceptibilidade do CIV convencional ao manchamento mediante imersão em soluções corantes, como substâncias pigmentantes provenientes da dieta, medicamentos na forma líquida, géis fluoretados, géis clareadores e soluções evidenciadoras de biofilme, além de corantes laboratoriais. Em contrapartida, alguns trabalhos constataram estabilidade de cor do CIV convencional em comparação a outros materiais restauradores. Conclusões: O CIV convencional apresenta estabilidade de cor variável, dependendo das soluções corantes e métodos de experimento aplicados.
\end{abstract}

Palavras-chave: Cimento de ionômero de vidro; Corantes; Odontologia; Estética.

\begin{abstract}
Background: Glass ionomer cement (GIC) has several indications in Dentistry, due to its biocompatibility properties, fluoride release and adhesion to dental tissues. Objective: To analyze the color stability of conventional GICs when immersed in coloring solutions based on data obtained in the literature. Methods: This is an integrative literature review carried out through searches in the databases PubMed, LILACS, SciELO and Cochrane Library, in which the following descriptors were used: "glass ionomer cement", "cimentos de ionômeros de vidro", "color", "discoloration", "pigmentação" e "cor". Eligibility criteria included studies that evaluated the color stability of conventional GICs, full articles available for download, and texts published in English, Portuguese and Spanish. Clinical trials, laboratory studies and in situ studies were included and studies published in the last 25 years. Results: After analyzing the articles, 14 studies were included in the review. Predominantly, conventional GICs susceptibility to staining has been reported by immersion in coloring solutions, such as pigmenting substances from the diet, drugs in liquid form, fluoride gels, bleaching gels and biofilm-evidence solutions, in addition to laboratory dyes. On the other hand, some studies have found the color stability of conventional GICs compared to other restorative materials. Conclusions: Conventional GIC has variable color stability, depending on the dye solutions and experimental methods applied.
\end{abstract}

Keywords: Glass ionomer cements; Coloring Agents; Dentistry; Esthetics. 


\begin{abstract}
Resumen
Introducción: El Cementos de Ionómero Vítreo (CIV) tiene varias indicaciones en Odontología, debido a sus propiedades de biocompatibilidad, liberación de flúor y adhesión a los tejidos dentarios. Objetivo: Analizar la estabilidad del color de los CIVs convencionales cuando se sumergen en soluciones colorantes con base en datos obtenidos en la literatura. Métodos: Esta es una revisión integrativa de la literatura realizada a través de búsquedas en las bases de datos PubMed, LILACS, SciELO y Cochrane Library, en las que se utilizaron los siguientes descriptores: "glass ionomer cement", "cimentos de ionômeros de vidro", "color", "discoloration", "pigmentação" e "cor". Los criterios de elegibilidad incluyeron estudios que evaluaron la estabilidad del color de los CIVs convencionales, artículos completos disponibles para descargar y textos publicados en inglés, portugués y español. Se incluyeron ensayos clínicos, estudios de laboratorio y estudios in situ y estudios publicados en los últimos 25 años. Resultados: Después del análisis de los artículos, se incluyeron 14 estudios en la revisión. Predominantemente, la susceptibilidad del CIV convencional a la tinción se ha informado por inmersión en soluciones colorantes, como sustancias pigmentarias de la dieta, medicamentos en forma líquida, geles de flúor, geles blanqueadores y soluciones de evidencia de biopelícula, además de colorantes de laboratorio. Por otro lado, algunos estudios han encontrado la estabilidad del color del CIV convencional en comparación con otros materiales restauradores. Conclusiones: El CIV convencional tiene una estabilidad de color variable, dependiendo de las soluciones de colorante y los métodos experimentales aplicados.

Palabras clave: Cementos de ionómero vítreo; Colorantes; Odontología; Estética.
\end{abstract}

\title{
1. Introdução
}

No contexto atual, a estética possui uma grande relevância para a Odontologia (Adusumilli et al., 2016). Diante disso, muitos materiais restauradores foram introduzidos no mercado a fim de atender às demandas funcionais e estéticas, além de buscar adequações às necessidades individuais apresentadas durante o tratamento. Dentre os materiais disponíveis, o cimento de ionômero de vidro (CIV) configura-se como uma boa alternativa, tendo em vista suas propriedades de liberação de flúor, baixo coeficiente de expansão térmica, biocompatibilidade e adesão à estrutura dentária (Bezgin, et al., 2015; Boscarioli, et al., 2002; Chakravarthy \& Clarence, 2018; Menezes-Silva et al., 2019; Prabhakar et al., 2013; Silva et al., 2021).

O CIV apresenta várias indicações clínicas, tais como proteção pulpar e forramento de cavidades, selamento de fóssulas e fissuras, cimentação de peças protéticas e bandas ortodônticas, núcleos de preenchimento, restaurações cervicais e o tratamento restaurador atraumático, bem como seu papel restaurador na dentição decídua devido às suas características anticariogênicas com grande utilidade na fase infantil (Choi et al., 2019; Diniz et al., 2021; Menezes-Silva et al., 2019; Sun et al., 2018; Sidhu \& Leal, 2019; Silva et al., 2021). A fim de melhorar as propriedades estéticas, resistência mecânica, manipulação e tempo de presa, algumas modificações foram realizadas no pó vítreo e no ácido reagente até as atuais formulações disponíveis do CIV (Almeida et al., 2021; Kale et al., 2019). Contudo, uma das principais fontes de insatisfação do paciente com restaurações de CIV diz respeito às diferenças visíveis na cor entre dentes e restaurações (Jafarpour et al., 2019).

A susceptibilidade do CIV à alteração de cor pode estar relacionada a fatores intrínsecos e extrínsecos. Os fatores intrínsecos são inerentes às características adversas do material, como tempo de presa, composição e quantidade de incorporação de água na restauração (Choi et al., 2019; Savas et al., 2019). Em contrapartida, os fatores extrínsecos incluem a adsorção e absorção de corantes resultante da contaminação por fontes exógenas provenientes da alimentação, hábitos do paciente, nicotina e algumas bebidas (Adusumilli et al., 2016; Choi et al., 2019; Kale et al., 2019; Prabhakar et al., 2013). Alterações na coloração de cimentos ionoméricos por soluções corantes, como café (Yildiz et al., 2015) e refrigerante de cola (Savas et al., 2019), e o manchamento após envelhecimento em diferentes soluções têm sido relatados na literatura (Choi et al., 2019).

Alguns estudos relatam que a alteração de cor no CIV pode ser influenciada por outros fatores, como ausência de proteção superficial nas primeiras 24 horas, rugosidade superficial do CIV, ausência de acabamento e polimento e ciclos de escovação (Bezgin et al., 2015; Yildiz et al., 2015). A partir disso, destaca-se a dinamicidade existente no ambiente oral e a necessidade de análises acerca da influência desses vários aspectos na estabilidade de cor do CIV. 
Nesse sentido, o objetivo do atual estudo consistiu em analisar a estabilidade de cor do cimento de ionômero de vidro convencional quando imerso em soluções corantes a partir de dados obtidos na literatura.

\section{Metodologia}

A pergunta que norteou o estudo foi: "há estabilidade de cor nos cimentos de ionômero de vidro convencionais quando imersos em soluções corantes?”. O estudo trata-se de uma revisão integrativa da literatura, que compreendeu os seguintes passos metodológicos: (1) identificação do problema e elaboração da pergunta de pesquisa; (2) pesquisa de estudos relevantes (estabelecimento das palavras-chaves, critérios de inclusão e exclusão, busca nas bases de dados); (3) seleção dos estudos baseada nos critérios de elegibilidade; (4) análise e elaboração de dados; e (5) apresentação dos resultados (Whittemore \& Knafl, 2005).

A busca foi realizada em 28 de setembro de 2021, por meio do portal BVS e das bases de dados PubMed, LILACS, SciELO e Cochrane Library. Os termos utilizados como descritores para as buscas foram: "glass ionomer cement", "cimentos de ionômeros de vidro", "color", "discoloration", "pigmentação" e "cor". Para as bases de dados PubMed e Cochrane Library foram utilizadas as estratégias de busca "glass ionomer cement AND color"e "glass ionomer cement AND discoloration". A estratégia "cimentos de ionômeros de vidro AND pigmentação" foi utilizada na BVS e LILACS. Na SciELO, foi usada a estratégia de busca "cimento de ionômero de vidro AND cor". A busca foi realizada através do título, resumo e palavras-chave.

\subsection{Critérios de elegibilidade dos artigos}

Os critérios de inclusão para seleção dos artigos foram: estudos que avaliaram a estabilidade de cor de CIVs convencionais, artigos completos e disponíveis para download, artigos publicados nos últimos 25 anos ao período atual, além dos textos escritos nos idiomas inglês, português e espanhol. Foram incluídos ensaios clínicos, estudos laboratoriais e estudos in situ.

Revisões de literatura, artigos repetidos e que avaliassem a estabilidade de cor de CIVs especializados (modificados por resina, CIVs de alta viscosidade e alterações dos componentes) foram excluídos desta revisão.

\subsection{Seleção dos estudos e extração dos dados}

A avaliação inicial foi feita através da leitura do título e do resumo, e selecionados com base nos critérios de elegibilidade. Após a seleção inicial, foram excluídos todos os artigos que não abordaram o tema repetidos, aqueles que não contemplaram os descritores no título e/ou resumo e removidos todos aqueles repetidos nas cinco bases de dados. Por fim, os artigos pré-selecionados foram lidos na íntegra por dois autores devidamente calibrados para determinar a amostra final e construir tabelas com as informações mais relevantes dos artigos selecionados. Dois autores realizaram a leitura completa dos artigos e a extração de dados. Os dados extraídos foram agrupados em uma planilha do Excel.

A partir das buscas foram identificados 836 artigos, após aplicar os critérios de inclusão e exclusão e remoção das duplicatas restaram 32 estudos, dos quais foram lidos os títulos e os resumos. Após essa leitura foram excluídos 12, restando 20 estudos que foram lidos na íntegra. Após a leitura na íntegra, foram eleitos 14 artigos que passaram a compor a amostra, conforme fluxograma construído seguindo a metodologia Preferred Reporting Items for Systematic Reviews and MetaAnalyses PRISMA ((Moher et al, 2009) apresentado na Figura 1. 
Figura 1. Fluxograma detalhando o processo de seleção dos artigos incluídos.

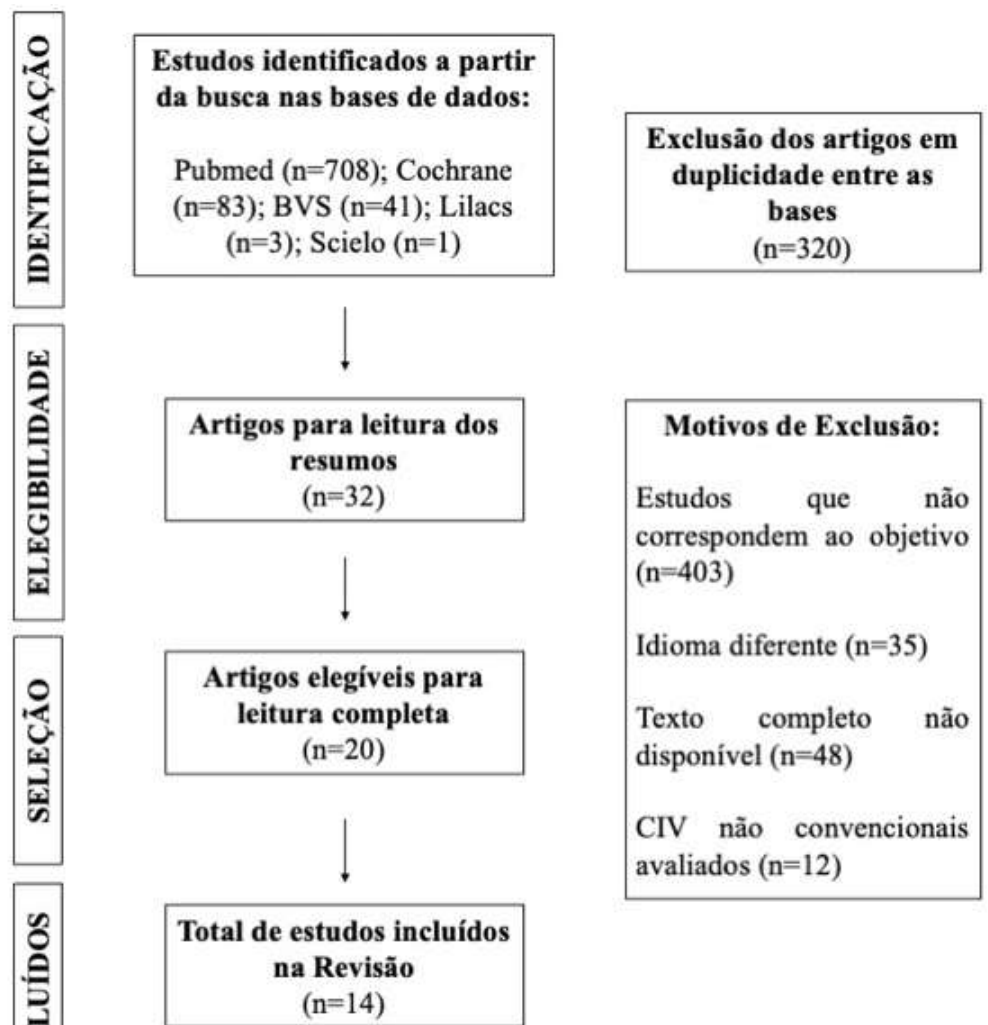

Fonte: Autores (2022).

\section{Resultados}

Após a aplicação dos critérios de inclusão e exclusão previamente organizados, foram incluídos 14 artigos. Com o propósito de sistematizar os dados dos artigos selecionados, no Quadro 1 estão representados: nome dos autores, ano de publicação, tamanho amostral de CIV convencional, as soluções corantes utilizadas, os grupos de materiais restaurados para comparação, os tempos e métodos de avaliação, além dos principais resultados. 
Research, Society and Development, v. 11, n. 3, e2511326190, 2022

(CC BY 4.0) | ISSN 2525-3409 | DOI: http://dx.doi.org/10.33448/rsd-v11i3.26190

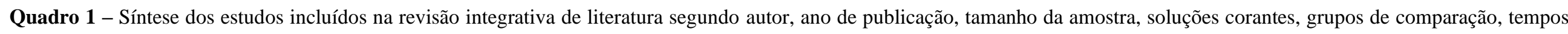
de avaliação, métodos de avaliação de cor e principais resultados.

\begin{tabular}{|c|c|c|c|c|c|c|}
\hline $\begin{array}{c}\text { Autor/ano de } \\
\text { publicação }\end{array}$ & $\begin{array}{c}\text { Tamanho da } \\
\text { amostra }\end{array}$ & Soluções Corantes & Grupos de Comparação & Tempos de avaliação & $\begin{array}{l}\text { Método de } \\
\text { avaliação }\end{array}$ & Principais resultados \\
\hline $\begin{array}{l}\text { Adusumilli et } \\
\text { al., } 2016\end{array}$ & $\begin{array}{l}100 \text { corpos de } \\
\text { prova }\end{array}$ & $\begin{array}{l}\text { Refrigerante de cola, } \\
\text { sorvete de laranja, } \\
\text { energético e água }\end{array}$ & $\begin{array}{l}\text { CIV convencional e } \\
\text { Giomer }\end{array}$ & $\begin{array}{l}\text { Imersão } 1,5 \text { ou } 10 \text { vezes } \\
\text { por } 5 \text { minutos, intervalos } \\
\text { de } 12 \text { horas durante } 15 \\
\text { dias }\end{array}$ & $\begin{array}{l}\text { Espectrofotômetro } \\
\text { (sistema } \\
\text { CIEL*a*b*) }\end{array}$ & $\begin{array}{l}\text { CIV apresentou alteração de cor significativa }(p<0,001) \\
\text { comparado ao Giomer quando imerso em refrigerante de cola } \\
\text { no maior tempo de imersão. Exceto a água, todas as soluções } \\
\text { produziram alteração de cor }\end{array}$ \\
\hline $\begin{array}{l}\text { Bezgin et al., } \\
2015\end{array}$ & $\begin{array}{l}40 \text { corpos de } \\
\text { prova }\end{array}$ & $\begin{array}{l}\text { Suco de cereja, } \\
\text { refrigerante de cola, } \\
\text { leite achocolatado e } \\
\text { água }\end{array}$ & $\begin{array}{l}\text { CIV convencional, } \\
\text { Compômero (CP) e } \\
\text { Resina Composta (RC) }\end{array}$ & $\begin{array}{l}\text { Imediato, } 1,7,30 \text { e } 60 \\
\text { dias }\end{array}$ & $\begin{array}{l}\text { Colorímetro } \\
\text { (sistema } \\
\text { CIEL*a*b*) }\end{array}$ & $\begin{array}{l}\text { Mudanças de cor foram significativamente maiores para a RC } \\
\text { do que para o CIV ou CP }(p<0,006) \text {. Todas as soluções } \\
\text { produziram alteração de cor, inclusive a água }\end{array}$ \\
\hline Fay et al., 1999 & $\begin{array}{l}20 \text { corpos de } \\
\text { prova }\end{array}$ & $\begin{array}{l}\text { Suco de uva, chá } \\
\text { instantâneo, } \\
\text { clorexidina e água }\end{array}$ & $\begin{array}{l}\text { CIV convencional, } \mathrm{CP} \text { e } \\
\text { RC }\end{array}$ & Imediato e 5 dias & $\begin{array}{l}\text { Espectrofotômetro } \\
\text { (sistema } \\
\text { CIEL*a*b*) }\end{array}$ & $\begin{array}{l}\text { CIV apresentou maior alteração de cor em todas as soluções } \\
\text { comparado a RC e ao CP, principalmente quando imerso em } \\
\text { suco de uva e chá. Mudanças de cor causadas pela água e } \\
\text { clorexidina não foram perceptíveis clinicamente }\end{array}$ \\
\hline $\begin{array}{l}\text { Chakravarthy \& } \\
\text { Clarence, } 2018\end{array}$ & $\begin{array}{l}10 \text { corpos de } \\
\text { prova }\end{array}$ & Vinho e água & $\begin{array}{l}\text { CIV convencional, } \mathrm{RC} \text { e } \\
\text { porcelana feldspática }\end{array}$ & $\begin{array}{l}\text { Imediata e pós-imersão. } \\
\text { Imersão por } 20 \text { minutos } \\
\text { durante } 28 \text { dias }\end{array}$ & $\begin{array}{l}\text { Espectrofotômetro } \\
\text { (sistema } \\
\text { CIEL*a*b*) }\end{array}$ & $\begin{array}{l}\text { CIV apresentou maior alteração de cor após imersão ao vinho } \\
\text { em comparação a RC e à porcelana }\end{array}$ \\
\hline $\begin{array}{l}\text { Jafarpour et al., } \\
2019\end{array}$ & $\begin{array}{l}72 \text { corpos de } \\
\text { prova }\end{array}$ & $\begin{array}{l}\text { Ácido lático, café e } \\
\text { água }\end{array}$ & $\begin{array}{l}3 \text { CIV convencional e } 2 \\
\text { CIV modificado por resina } \\
\text { (CIVMR) cobertos ou não } \\
\text { com RC }\end{array}$ & 24 horas e 7 dias & $\begin{array}{l}\text { Espectrofotômetro } \\
\text { (sistema } \\
\text { CIEL*a*b*) }\end{array}$ & $\begin{array}{l}\text { Alteração de cor no CIV convencional após } 7 \text { dias de imersão } \\
\text { em todas as soluções (inclusive em água) independente da } \\
\text { cobertura de resina. Foi observada diferença significativa } \\
(p<0,05) \text { no manchamento do café no CIV convencional não } \\
\text { cobertos por resina. Porém, a maior alteração de cor ocorreu } \\
\text { com o CIVMR }\end{array}$ \\
\hline Pani et al., 2020 & $\begin{array}{l}20 \text { corpos de } \\
\text { prova }\end{array}$ & $\begin{array}{l}\text { Mistura de café, } \\
\text { refrigerante de cola e } \\
\text { suco de uva em iguais } \\
\text { proporções }\end{array}$ & $\begin{array}{l}\text { CIV convencional, CIV } \\
\text { reforçados com nanotubos } \\
\text { de carbono e } \\
\text { nanopartículas de prata }\end{array}$ & $\begin{array}{l}\text { Imediato, } 1 \text { hora, } 24 \text { horas } \\
\text { e } 1 \text { semana }\end{array}$ & $\begin{array}{l}\text { Espectrofotômetro } \\
\text { (sistema } \\
\text { CIEL*a*b*) }\end{array}$ & $\begin{array}{l}\text { CIV convencional apresentou menor alteração de cor quando } \\
\text { comparado aos CIVs reforçados testados em todos os tempos } \\
\text { de avaliação }\end{array}$ \\
\hline Kale et al., 2019 & $\begin{array}{l}40 \text { corpos de } \\
\text { prova }\end{array}$ & $\begin{array}{l}\text { Amoxicillina + ácido } \\
\text { clavulânico, } \\
\text { metronidazol, } \\
\text { cefalexina, } \\
\text { ibuprofeno, } \\
\text { ibuprofeno } \\
\text { paracetamol }\end{array}$ & $\begin{array}{l}\text { CIV convencional, } \mathrm{RC} \text { e } \\
\text { CIV reforçado com } \\
\text { zircônia }\end{array}$ & $\begin{array}{l}\text { Imediato, pós-ciclagens } \\
\text { de pH (desmineralização } \\
\text { por } 8 \text { horas e } \\
\text { remineralização por } 16 \\
\text { horas) e pós-imersão } \\
\text { (soluções sob agitação por } \\
2 \text { minutos a cada } 8 \text { horas } \\
\text { por } 1 \text { semana) }\end{array}$ & $\begin{array}{l}\text { Espectrofotômetro } \\
\text { (sistema } \\
\text { CIEL*a*b*) }\end{array}$ & $\begin{array}{l}\text { CIV convencional apresentou menor alteração de cor } \\
\text { estatisticamente significativa }(\mathrm{p}<0,05) \text { comparada a RC e } \\
\text { CIV reforçado por zircônia, exceto para a RC imersa em } \\
\text { amoxicilina e cefalexina. O CIV convencional apresentou } \\
\text { maior alteração de cor quando imerso na amoxicilina+ácido } \\
\text { clavulânico }\end{array}$ \\
\hline $\begin{array}{l}\text { Li et al., } 2009 \\
\text { (in situ) }\end{array}$ & $\begin{array}{l}38 \text { corpos de } \\
\text { prova }\end{array}$ & $\begin{array}{lr}\begin{array}{l}\text { Peróxido } \\
\text { carbamida }\end{array} & \text { a } \\
\text { controle } & \text { de } \\
\text { co } & \text { (sem }\end{array}$ & $\begin{array}{l}\text { CIV convencional, } 2 \text { tipos } \\
\text { de RC e CIVMR }\end{array}$ & $\begin{array}{l}\text { Imediato, } 1,2,3 \text { e } 4 \\
\text { semanas, e } 2 \text { semanas } \\
\text { após finalização } \quad \text { do }\end{array}$ & $\begin{array}{l}\text { Espectrofotômetro } \\
\text { (sistema } \\
\text { CIEL*a*b*) }\end{array}$ & $\begin{array}{l}\text { Todos os materiais apresentaram alteração de cor quando } \\
\text { submetidos ao agente clareador, principalmente nas duas } \\
\text { primeiras semanas, inclusive o CIV. Após a retirada do }\end{array}$ \\
\hline
\end{tabular}


Research, Society and Development, v. 11, n. 3, e2511326190, 2022

(CC BY 4.0) | ISSN 2525-3409 | DOI: http://dx.doi.org/10.33448/rsd-v11i3.26190

\begin{tabular}{|c|c|c|c|c|c|c|}
\hline & & tratamento clareador) & & $\begin{array}{l}\text { clareamento. } \text { Aplicação } \\
\text { do clareador } 8 \text { horas por } \\
\text { dia, durante } 4 \text { semanas }\end{array}$ & & $\begin{array}{l}\text { peróxido de carbamida, o CIV convencional apresentou } \\
\text { diminuição da alteração de cor até uma faixa clinicamente } \\
\text { aceitável }\end{array}$ \\
\hline $\begin{array}{l}\text { Wang \& Huang, } \\
2014\end{array}$ & $\begin{array}{l}70 \text { corpos de } \\
\text { prova }\end{array}$ & $\begin{array}{l}\text { Flúor } r \text { fosfato } \\
\text { acidulado gel em } \\
\text { concentrações } \quad(4.07, \\
8.13, \quad 16.26, \quad 32.52, \\
65.04 \text { e } 100) \text { e água }\end{array}$ & CIV convencional & $\begin{array}{l}\text { Aplicação dos géis por } 4 \\
\text { minutos, lavagem e } \\
\text { estocagem. Avaliação } 24 \\
\text { horas da primeira } \\
\text { aplicação e } 24 \text { horas da } \\
\text { segunda aplicação }\end{array}$ & $\begin{array}{l}\text { Escala de cor pelo } \\
\text { método visual }\end{array}$ & $\begin{array}{l}\text { O CIV apresentou alteração de cor observada visualmente, } \\
\text { sendo associada com a concentração e frequência de } \\
\text { aplicação do flúor, além do aumento da acidez do gel } \\
\text { fluoretado }\end{array}$ \\
\hline $\begin{array}{l}\text { Debner et al., } \\
2000\end{array}$ & $\begin{array}{l}5 \text { corpos de } \\
\text { prova }\end{array}$ & $\begin{array}{l}3 \text { vernizes fluoretados } \\
\text { e água }\end{array}$ & $\begin{array}{l}\text { CIV convencional, } \mathrm{CP} \text { e } \\
\text { RC }\end{array}$ & $\begin{array}{l}\text { Imediato e } 4 \text { horas após } \\
\text { aplicação dos vernizes }\end{array}$ & $\begin{array}{l}\text { Espectrofotômetro } \\
\text { (sistema } \\
\text { CIEL*a*b*) }\end{array}$ & $\begin{array}{l}2 \text { vernizes causaram alteração de cor perceptível em todos os } \\
\text { materiais, inclusive no CIV convencional }\end{array}$ \\
\hline $\begin{array}{l}\text { Prabhakar et al., } \\
2013\end{array}$ & $\begin{array}{l}20 \text { corpos de } \\
\text { prova }\end{array}$ & Água & $\begin{array}{lr}\text { CIV } & \text { convencional, } \\
\text { CIVMR, } & \text { CIV } \\
\text { convencional e CIVMR } \\
\text { associados a } 1.25 \%, \text { e } \\
2.5 \% \text { de Diacetato de } \\
\text { Clorexidina }\end{array}$ & 24 horas, 7 dias e 30 dias & $\begin{array}{l}\text { Espectrofotômetro } \\
\text { (sistema } \\
\text { CIEL*a*b*) }\end{array}$ & $\begin{array}{l}\text { CIV convencional apresentou menor alteração de cor do que } \\
\text { o CIVMR. Não foi observada mudança significativa na o } \\
\text { CIV convencional e os CIVs associados a clorexidina. Não } \\
\text { houve alteração de cor significativa entre } 7 \text { dias e } 1 \text { mês em } \\
\text { todos os CIVs testados }\end{array}$ \\
\hline $\begin{array}{l}\text { Boscarioli et al., } \\
2002\end{array}$ & $\begin{array}{l}40 \text { corpos de } \\
\text { prova }\end{array}$ & $\begin{array}{l}\text { Solução aquosa de } \\
\text { azul de metileno a } 2 \%\end{array}$ & $\begin{array}{l}\text { CIV convencional, } 2 \text { tipos } \\
\text { de CIVMR }\end{array}$ & $\begin{array}{l}3 \text { minutos de aplicação } \\
\text { (concentração do corante } \\
\text { após trituração das } \\
\text { amostras em } 48 \text { horas em } \\
\text { solução alcoólica) }\end{array}$ & $\begin{array}{l}\text { Espectrofotômetro } \\
\text { (curva de } \\
\text { absorbância) }\end{array}$ & $\begin{array}{l}\text { CIV convencional apresentou maior concentração de corante } \\
\text { comparado ao CIVMR }\end{array}$ \\
\hline $\begin{array}{l}\text { Pimenta et al., } \\
1997\end{array}$ & $\begin{array}{l}47 \text { corpos de } \\
\text { prova }\end{array}$ & $\begin{array}{l}\text { Verde de malaquita e } \\
\text { fucsina básica a } 0,5 \%\end{array}$ & 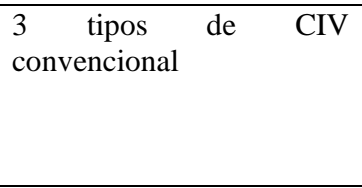 & $\begin{array}{l}1 \text { minuto de aplicação } \\
\text { (concentração do corante } \\
\text { após trituração das } \\
\text { amostras em } 24 \text { horas em } \\
\text { solução alcoólica) }\end{array}$ & $\begin{array}{l}\text { Espectrofotômetro } \\
\begin{array}{l}\text { (curva de } \\
\text { absorbância) }\end{array}\end{array}$ & $\begin{array}{l}\text { Todos os CIVs apresentaram concentração de corante, mas o } \\
\text { CIV com ácido polimaleico apresentou menor manchamento } \\
\text { do que os CIVs com ácido poliacrílico na composição. A } \\
\text { fucsina básica apresentou um grau de manchamento maior no } \\
\text { CIV convencional }\end{array}$ \\
\hline $\begin{array}{l}\text { Saito et al., } \\
2000\end{array}$ & $\begin{array}{l}60 \text { corpos de } \\
\text { prova }\end{array}$ & $\begin{array}{l}\text { Fucsina básica } 0,5 \% \text { e } \\
\text { eritrosina } 0,5 \%\end{array}$ & $\begin{array}{l}\text { CIV convencional, } \mathrm{CP} \text { e } \\
\text { CIVMR }\end{array}$ & $\begin{array}{l}24 \text { horas de imersão } \\
\text { (concentração do corante } \\
\text { após trituração das } \\
\text { amostras em } 24 \text { horas em } \\
\text { solução alcoólica) }\end{array}$ & \begin{tabular}{l}
\multicolumn{2}{l}{ Espectrofotômetro } \\
(curva de \\
absorbância)
\end{tabular} & $\begin{array}{l}\text { CIV convencional apresentou maior deposição de corante } \\
\text { comparado ao CP e CIVMR. A fucsina pigmentou mais que a } \\
\text { eritrosina }\end{array}$ \\
\hline
\end{tabular}

Fonte: Autores (2022). 


\section{Discussão}

Os materiais restauradores estão constantemente expostos a variados desafios no microambiente oral, o que resulta em uma diversidade de alterações nas suas propriedades físicas, mecânicas e estéticas no decorrer do tempo. Nesse contexto, a estabilidade de cor influencia na longevidade e durabilidade das restaurações, levando a necessidade de substituições (Kale et al., 2019).

A maioria dos trabalhos incluídos nesta revisão relatam a susceptibilidade do CIV convencional ao manchamento mediante imersão em variadas soluções corantes e protocolos experimentais (Adusumilli et al., 2016; Boscarioli et al., 2002; Chakravarthy \& Clarence, 2018; Debner et al., 2000; Fay et al., 1999; Jafarpour et al., 2019; Pimenta et al., 1997; Saito et al., 2000; Wang \& Huang, 2014). Em contrapartida, alguns artigos da presente revisão observaram estabilidade de cor do CIV convencional em comparação a outros materiais restauradores (Bezgin et al., 2015; Kale et al., 2019; Li et al., 2009; Pani et al., 2020; Prabhakar et al., 2013).

Estudos mostram que o potencial para manchamento do CIV vem sendo relacionado a diversos fatores intrínsecos, adsorção física e reações físico-químicas inerentes ao material (Adusumilli et al., 2016; Chakravarthy \& Clarence, 2018; Jafarpour et al., 2019). A sinérese, caracterizada pela perda de água do CIV para o meio, torna a sua superfície fraca e opaca, com subsequentes fissuras que facilitam a penetração de pigmentos (Adusumilli et al., 2016). Do mesmo modo, a sorção de água do CIV pode influenciar na alteração de cor (Fay et al., 1999). Segundo Bezgin et al. (2015), o manchamento no CIV após a imersão em soluções corantes por períodos longos (a partir de 1 mês) podem ser atribuídas à absorção higroscópica do material.

Outros fatores como porosidade das partículas de vidro, conteúdo de poliácido e degradação de sais de poliacrilato de metal, bem como a presença de microfissuras podem influenciar na estabilidade de cor do CIV (Chakravarthy \& Clarence, 2018). Alguns autores sugerem que o acabamento e polimento superficial do CIV pode torná-lo mais resistente ao manchamento (Fay et al., 1999; Boscarioli et al., 2002), uma vez que as irregularidades na superfície podem contribuir para o acúmulo de substâncias corantes (Saito et al., 2000). No estudo in situ de Li et al. (2009), as fotomicrografias apresentaram mudanças morfológicas do CIV submetido a géis clareadores, sendo observado rugosidade superficial significativamente aumentada e fissuras extensas. No entanto, as alterações de cor do CIV convencional foram menores em relação a estudos anteriores, o que pode ser atribuído às trocas iônicas entre a saliva e o cimento (Li et al., 2009).

Para tanto, o CIV deve ser protegido da contaminação salivar e exposição aos corantes, seja por agentes de proteção ou resinas de cobertura (Adusumilli et al., 2016). Os agentes protetores de superfície do CIV geralmente são vernizes caracterizados por natureza hidrofóbica que podem oferecer dificuldade de umedecimento pela substância corante (Boscarioli et al., 2002), devido às diferenças de tensão superficial. Debner et al. (2000) relataram manchamento no CIV perceptível após exposição a dois tipos dos vernizes fluoretados avaliados.

Por outro lado, a maior estabilidade de cor do CIV convencional foi observada experimentalmente em vários trabalhos (Bezgin et al., 2015; Kale et al., 2019; Li et al., 2009; Pani et al., 2020; Prabhakar et al., 2013). A maior resistência às alterações de cor do CIV pode ser atribuída à capacidade de pigmentação das soluções corantes e às propriedades inerentes ao material, destacando-se a sorção de água (Bezgin et al., 2015) e as características das partículas de vidro do CIV (Kale et al., 2019). O CIV convencional, caracterizado como material hidrofílico é menos susceptível ao manchamento uma vez que seu alto teor de água permite que eles absorvam menos água (Bezgin et al., 2015; Kale et al., 2019). No estudo de Jafarpour et al. (2013), o CIV convencional apresentou menor sorção de água quando comparado ao CIVMR. Ademais, as partículas de vidro, que apresentam o conteúdo inorgânico e que representam o núcleo do CIV, não permitem absorção de corantes na maior parte do material (Bezgin et al., 2015; Kale et al., 2019); embora alguma água possa ser absorvida através da superfície e da matriz orgânica mais superficial do CIV (Kale et al., 2019). 
Além disso, o potencial de pigmentação da solução corante pode influenciar no manchamento do CIV, dependendo particularmente do tamanho de partícula do agente de coloração, bem como a natureza hidrofílica ou hidrofóbica do mesmo (Kale et al., 2019). Para avaliação da estabilidade de cor, os estudos simularam o contato do CIV com soluções corantes que compõem a dieta, medicamentos utilizados na forma líquida, soluções usadas em procedimentos odontológicos, como géis fluoretados, géis clareadores e soluções evidenciadoras de biofilme, além de corantes laboratoriais.

Adusumilli et al. (2016) destacaram que o manchamento do CIV imerso em refrigerante de cola pode ser devido ao baixo $\mathrm{pH}$, e subsequente alteração na superfície e adsorção dos pigmentos. No entanto, no estudo de Bezgin et al. (2015), o meio ácido usado (cola e suco de cereja) não causou maior coloração do CIV em relação à resina composta.

É importante mencionar algumas limitações da presente revisão integrativa, tais como a não utilização de um instrumento para análise da qualidade dos artigos e interpretação dos resultados, além da restrição dos idiomas e ausência de busca da literatura "cinzenta". Vale ressaltar alguns aspectos fundamentais que correspondem ao protocolo de manipulação e que contribuem na estabilidade da cor do CIV a longo prazo, sendo eles a correta proporção de pó e líquido, tempo de manipulação, preparo e inserção na cavidade, proteção da superfície, além do acabamento e polimento.

\section{Conclusão}

Mediante as evidências expostas na atual revisão, pode-se concluir o cimento de ionômero de vidro convencional apresenta estabilidade de cor variável, dependendo das soluções corantes e método de experimento empregados.

A partir da leitura dos artigos, sugere-se a realização de estudos futuros para avaliação da estabilidade de cor do CIV e a associação com variáveis do microambiente oral, tais como variações de temperatura e pH, esforços mastigatórios, simulação de escovação e desafio cariogênico.

\section{Referências}

Adusumilli, H., Avula, J. S. S., Kakarla, P., Bandi, S., Mallela, G. M. K. \& Vallabhaneni, K (2016). Color stability of esthetic restorative materials used in pediatric dentistry: an in vitro study. Journal of the Indian Society of Pedodontics and Preventive Dentistry, 34(3), 233-237. https://doi.org/10.4103/09704388.186740

Almeida, M. B. de, Santos, P. H. dos, Esteves, L. M. B., Silva, L. M. A. V. da, Gallinari, M. de O., Fagundes, T. C., Antonnaccio, G. B. de M. \& Briso, A. L. F. (2021). Efeito da contaminação e limpeza da dentina na resistência de união do cimento de ionômero de vidro modificado por resina. Research, Society and Development, 10(6), e53310615983. https://doi.org/10.33448/rsd-v10i6.15983

Bezgin, T., Özer, L., Tulga Öz, F. \& Özkan, P. (2015). Effect of toothbrushing on color changes of esthetic restorative materials. Journal of Esthetic and Restorative Dentistry, 27(Suppl 1), S65-S73. https://doi.org/10.1111/jerd.12136.

Boscarioli, A. P. T., Lovadino, J. R., Consani, S. \& Goes, M. F. (2002). Influência da proteção e acabamento superficial sobre a resistência ao manchamento de cimentos ionoméricos restauradores. PGR - Pós-Graduação Revista da Faculdade de Odontologia de São José dos Campos, 5(1), 69-77.

Chakravarthy, Y. \& Clarence, S. (2018). The effect of red wine on colour stability of three different types of esthetic restorative materials: an in vitro study. Journal of Conservative Dentistry, 21(3), 319-323. https://doi.org/10.4103/JCD.JCD_293_17.

Choi, J. W., Lee, M. J., Oh, S. H. \& Kim, K. M. (2019). Changes in the physical properties and color stability of aesthetic restorative materials caused by various beverages. Dental Materials Journal, 38(1), 33-40. https://doi.org/ 10.4012/dmj.2017-247

Debner, T., Warren, D. P. \& Powers, J. M. (2000). Effects of fluoride varnish on color of esthetic restorative material. The International Journal of Esthetic Dentistry, 12, 160-163.

Diniz, A. C. S., Couto, G. A. do, Silva, T. B. da, Bauer, J. R. \& Firoozmand, L. M. (2021). Comportamento clínico de ionômero de vidro bioativo (45S5) em lesões de cárie moderada: Protocolo de estudo de um ensaio clínico. Research, Society and Development, 10(10), e07101018190. https://doi.org/10.33448/rsdv10i10.18190

Fay, R. M., Servos, T. \& Powers, J. M. (1999). Color of restorative materials after staining and bleaching. Operative Dentistry, $24,292-296$.

Jafarpour, D., Mese, A., Ferooz, M. \& Bagheri, R. (2019). The effects of nanofilled resin-based coatings on the physical properties of glass ionomer cement restorative materials. Journal of Dentistry, 89, 10317. https://doi.org/10.1016/j.jdent.2019.07.015.

Kale, Y. J., Nalwade, A. V., Dahake, P. T., Dapde, M. V. \& Kendre, S. B. (2019) Effect of different pediatric drug formulations on color stability of composite, zirconia-reinforced glass ionomer cement, and glass ionomer cement. Journal of the Indian Society of Pedodontics and Preventive Dentistry, 37 , 151-156. https://doi.org/10.4103/JISPPD.JISPPD_280_18. 
Research, Society and Development, v. 11, n. 3, e2511326190, 2022

(CC BY 4.0) | ISSN 2525-3409 | DOI: http://dx.doi.org/10.33448/rsd-v11i3.26190

Li, Q., Yu, H. \& Wang, Y. (2009). Colour and surface analysis of carbamide peroxide bleaching effects on the dental restorative materials in situ. Journal of Dentistry, 37, 348-356. https://doi.org/10.1016/j.jdent.2009.01.003.

Menezes-Silva, R., Cabral, R. N., Pascotto, R. C., Borges, A. F. S., Martins, C. C., Navarro, M. F. L., Sidhu, S. K. \& Leal, S. C. (2019). Mechanical and optical properties of conventional restorative glass-ionomer cements - a systematic review. Journal of Applied Oral Science, 21(27), e2018357. https://doi.org/ $10.1590 / 1678-7757-2018-0357$

Moher, D., Liberati, A., Tetzlaff, J., Altman, D. G. \& Group, P. (2009). Preferred reporting items for systematic reviews and meta-analyses: the PRISMA statement. PLoS Medicine, 6(7), e1000097. https://doi.org/10.1371/journal.pmed.1000097.

Pani, S. C., Aljammaz, M. T., Alrugi, A. M., Aljumaah, A. M., Alkahtani, Y. M. \& AlKhuraif, A. (2020). Color stability of glass ionomer cement after reinforced with two different nanoparticles. International Journal of Dentistry, 31, 7808535. https://doi.org/10.1155/2020/7808535.

Pimenta, I. C., Lovadino, J. R., Pimenta, L. A. F., Paulillo, L. A. M. S. \& Liporoni, P. C. S. (1997). Avaliação do manchamento dos cimentos de ionômero de vidro por soluções evidenciadoras de placa dental. Revista ABO Nacional, 5(2), 110-112.

Prabhakar, A. R., Pattanshetti, K. \& Sugandhan, S. (2013). A comparative study of color stability and fluoride release from glass ionomer cements combined with chlorhexidine. International Journal of Clinical Pediatric Dentistry, 6(1), 26-29. https://doi.org/10.5005/jp-journals-10005-1181.

Saito, S. K., Lovadino, J. R. \& Kroll, L. B. (2000). Rugosidade e pigmentação superficial de materiais ionoméricos. Pesquisa Odontológica Brasileira, 14(4), 351-356.

Savas, S., Colgecen, O., Yasa, B. \& Kucukyilmaz E. (2019). Color stability, roughness, and water sorption/solubility of glass ionomer-Based restorative materials. Nigerian Journal of Clinical Practice, 22(6), 824-832. https://doi.org/10.5005/jp-journals-10005-1181.

Silva, D. O. C. da, Silva, I. de M., Rocha, A. de O., Anjos, L. M. dos, Lima, T. O., Santos, R. de M. dos A. \& Cruz, B. P. (2021). Cimento de ionômero de vidro e sua aplicabilidade na Odontologia: Uma revisão narrativa com ênfase em suas propriedades. Research, Society and Development, 10(5), e20110514884. https://doi.org/10.33448/rsd-v10i5.14884

Sun, L., Yan, Z., Duan, Y., Zhang, J. \& Liu, B. (2018). Improvement of the mechanical, tribological and antibacterial properties of glass ionomer cements by fluorinated graphene. Dental Materials, 34(6), e115-e127. https://doi.org/10.1016/j.dental.2018.02.006.

Wang, E. \& Huang, B. (2014). Discolouration of glass-ionomer cement at different fluoride concentration levels. Oral Health and Dental Management, 13(2), $1-4$.

Whittemore, R. \& Knafl, K. (2005) The integrative review: updated methodology. Journal of Advanced Nursing, 52(5), 546-553. https://doi.org/ $10.1111 / \mathrm{j} .1365-2648.2005 .03621 . \mathrm{x}$

Yildiz, E., Karaarslan, E. S., Simsek, M., Ozsevik, A. S. \& Usumez, A. (2015). Color stability and surface roughness of polished anterior restorative materials. Dental Materials Journal, 34(5), 629-639. https://doi.org/10.4012/dmj.2014-344. 